University of Nebraska - Lincoln

DigitalCommons@University of Nebraska - Lincoln

20th \& 21st Century French and Francophone Modern Languages and Literatures, Department Studies International Colloquium

3-16-2020

\title{
Terre brûlée : une éco-poétique du vide
}

Pierre Taminiaux

Georgetown University, taminiap@georgetown.edu

Follow this and additional works at: https://digitalcommons.unl.edu/ffsc2020

Part of the Art Practice Commons, Comparative Literature Commons, Fine Arts Commons, French and Francophone Literature Commons, Other French and Francophone Language and Literature Commons, and the Visual Studies Commons

Taminiaux, Pierre, "Terre brûlée : une éco-poétique du vide" (2020). 20th \& 21st Century French and Francophone Studies International Colloquium. 2.

https://digitalcommons.unl.edu/ffsc2020/2

This Article is brought to you for free and open access by the Modern Languages and Literatures, Department of at DigitalCommons@University of Nebraska - Lincoln. It has been accepted for inclusion in 20th \& 21st Century

French and Francophone Studies International Colloquium by an authorized administrator of DigitalCommons@University of Nebraska - Lincoln. 
Terre brûlée : une éco-poétique du vide

Pierre Taminiaux/Georgetown University

20th/21St Century French and Francophone Studies

Conference, University of Lincoln-Nebraska, 2020.

Au XXle siècle, les sociétés occidentales sont confrontées à de nouvelles formes de chaos. Celui-ci comporte plusieurs dimensions, à la fois sociales, culturelles et écologiques. L'art doit dès lors prendre en considération cette situation d'une manière ou d'une autre. C'est ce qui m'autorise à parler ici de mes images photographiques rassemblées sous le titre de Terre brûlée.

Celles-ci ont été prises à l'automne 2017, au Portugal, près de la ville de Leiria, dans une forêt de bord de mer (une pinède, plus exactement) qui fut longtemps considérée dans l'histoire de ce pays comme un domaine royal. Cette saison fut marquée par de violents incendies qui se propagèrent avec une grande vitesse et entrainèrent de nombreuses destructions. Des dizaines de personnes trouvèrent en outre la mort dans ces évènements.

Je traversai en voiture cette zone sinistrée sans avoir vraiment conscience au départ de l'étendue des dégâts. Je pus alors constater les effrayants ravages issus de cette catastrophe naturelle, qui avait embrasé la forêt entière et emporté tout sur son passage. II ne demeurait ainsi qu'une terre calcinée, un grand espace couvert de cendres et sans vie. La végétation ellemême, des branches aux feuilles d'arbres, avait pratiquement disparu dans la tourmente. 


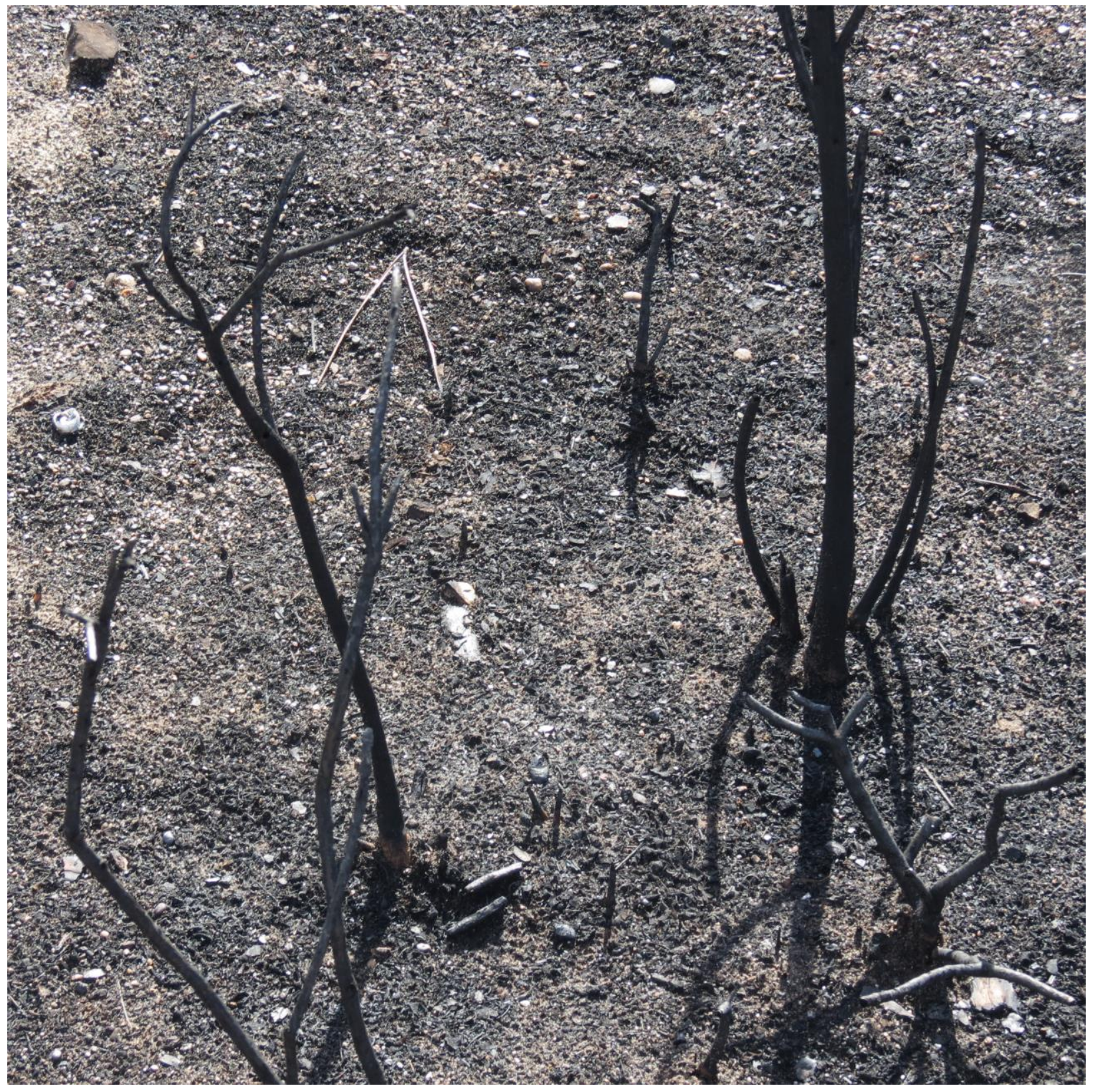




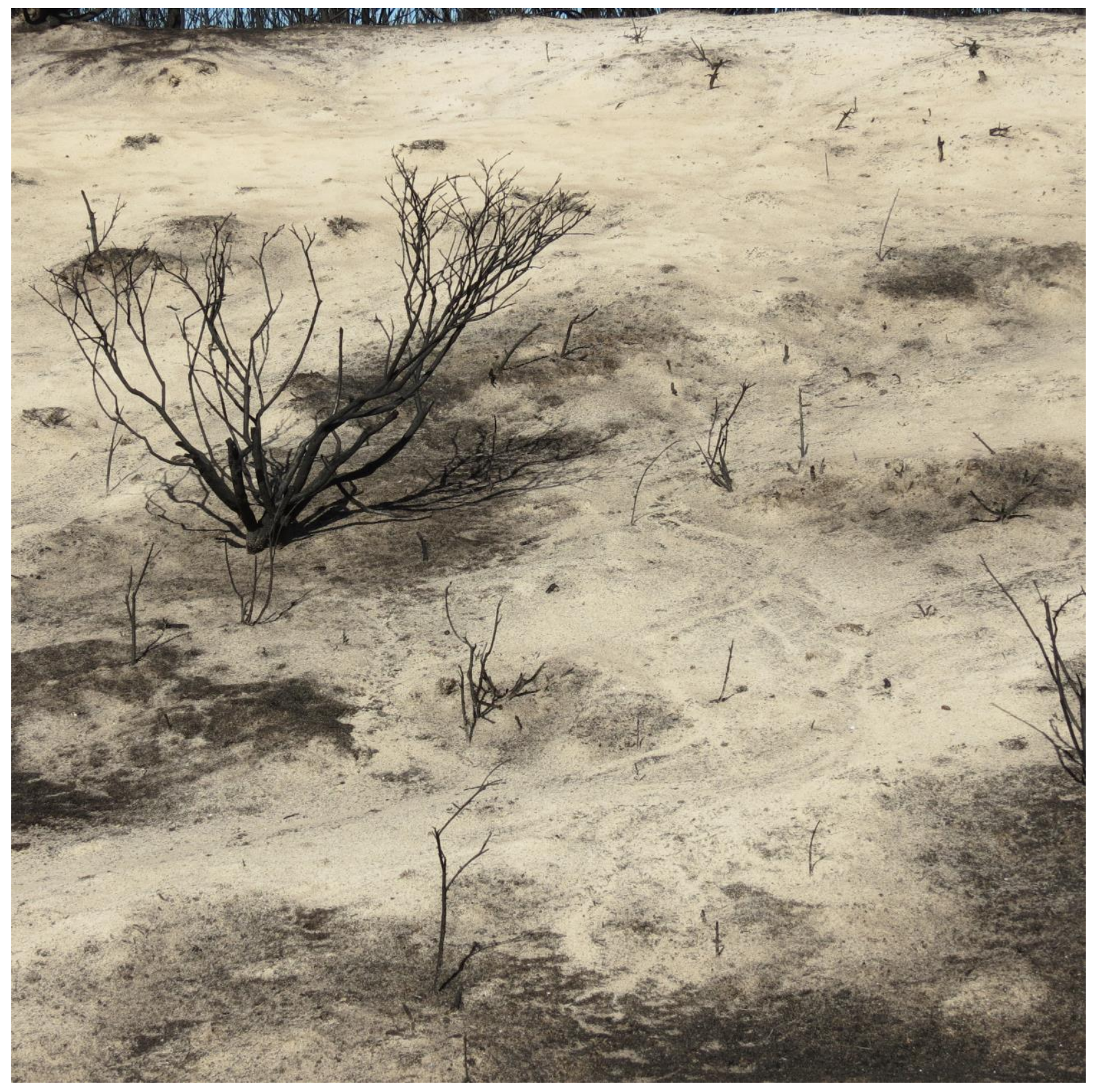




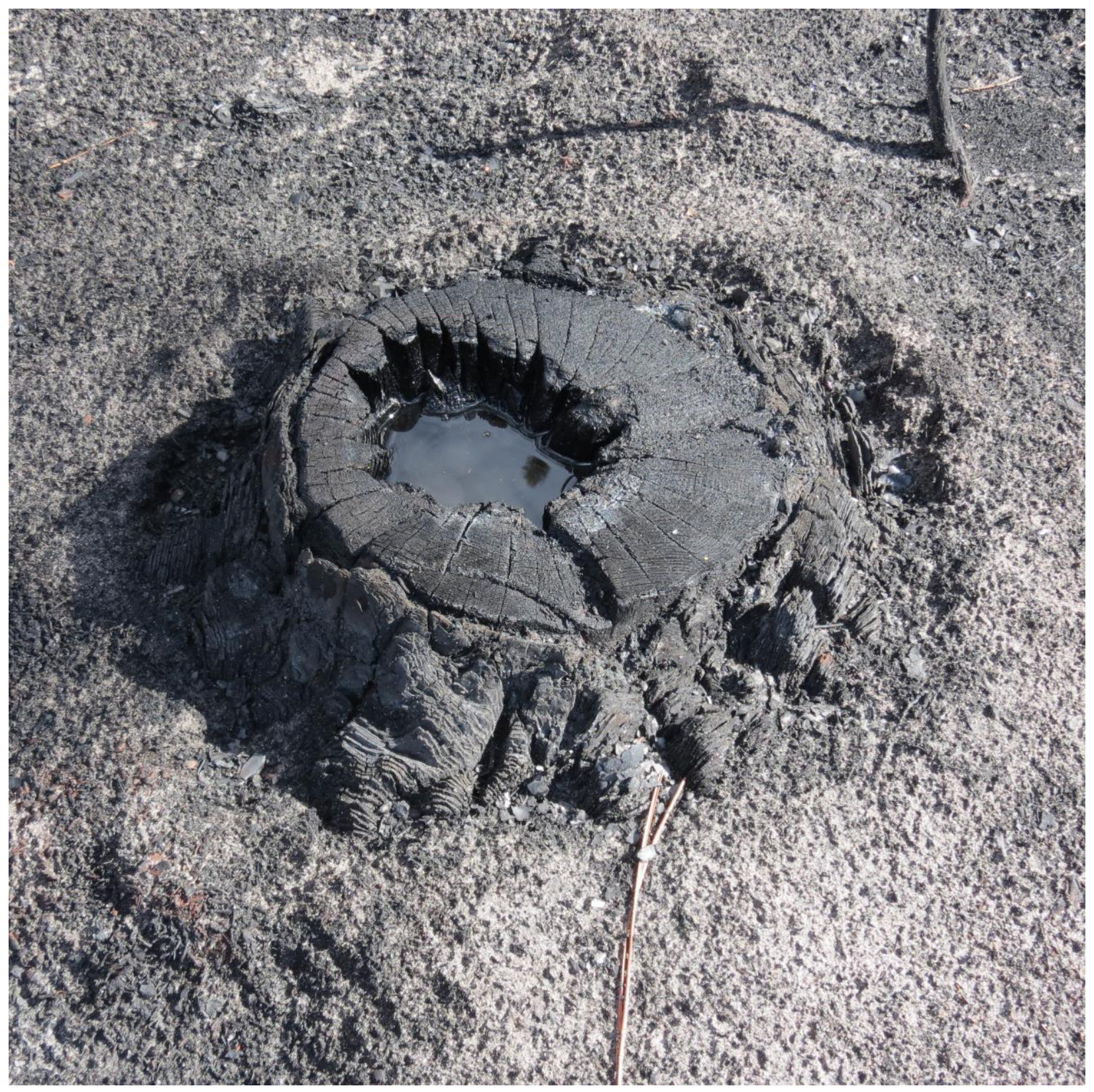


Le thème du vide renvoie certes à un parti-pris esthétique minimaliste et à une sorte de simplicité des formes. Mais il témoigne en outre d'une quête philosophique du silence et de la méditation dans un monde en permanence saturé d'images et de signes où ceux-ci sont de plus en plus difficiles à trouver ${ }^{1}$.

Ce vide fut au centre de certaines des démarches artistiques les plus novatrices du XXe siècle, de Marcel Duchamp à John Cage. Il ne fut pas non plus absent du surréalisme, si l'on songe en particulier à Nadja, livre parsemé de photographies des rues, des places, des statues et des bâtiments de Paris, et qui toutes donnent l'impression d'un espace urbain désert et comme désaffecté.

Le vide est à la fois porteur de vie et de mort. Les civilisations orientales ont insisté sur son aspect positif et transcendant, en montrant par exemple que ce vide pouvait mener l'homme à une expérience extatique de la réalité. Ce n'est pas un hasard, dans cette perspective, si de nombreux écrivains modernistes furent attirés et même plus inspirés par le bouddhisme zen, de Borges à Paz².

Dans Terre brûlée, la dimension de la destruction et du néant semble l'emporter cependant. Le vide est le résultat ici du pouvoir du feu, pouvoir d'annihilation radicale. II reflète les ténèbres du monde naturel, qui loin de fournir à l'humanité l'harmonie et la sérénité dont elle a besoin, apporte au contraire souvent le chaos et la désolation. Le XXIe siècle, faut-il le rappeler, est celui d'une transformation profonde des équilibres écologiques au niveau planétaire. Il a déjà donné naissance à cet égard à de nombreux cataclysmes, ouragans,

\footnotetext{
${ }^{1}$ Sur cette question, je renvoie à l'ouvrage Littératures et arts du vide, Paris : Hermann 2018, que j'ai codirigé avec Jérôme Duwa. Il est issu d'un colloque international de Cerisy qui s'est tenu en 2017.

${ }^{2}$ Voir à ce sujet l'ouvrage de Jorge Luis Borges et Alicia Jurado, Qu'est-ce que le Bouddhisme? Paris: Gallimard, 1979, ainsi que le poème de Paz 'Blanc', qui figure dans le recueil Versant Est, Paris: Gallimard, 1970.
} 
tsunamis, tremblements de terre, vagues de chaleur et sècheresses interminables à travers le monde.

Incontestablement, une telle évolution de la terre et de ses caractéristiques physiques entraîne de nouvelles attitudes vis-à-vis de l'environnement. Celui-ci est maintenant souvent perçu comme porteur de menaces pour notre propre survie et notre propre intégrité. Mais il s'agit bien de phénomènes globaux, et donc, en ce sens, de problèmes qui appartiennent à la communauté humaine toute entière.

L'art ne peut pas demeurer indiffèrent devant de tels bouleversements. 'La beauté sera convulsive ou ne sera pas', disait Breton en conclusion de Nadja. Notre monde actuel est ainsi rempli de convulsions et de chocs qui entrainent des traumatismes à la fois individuels et collectifs. C'est dans la nature que ces traumatismes s'inscrivent d'abord, et en particulier dans la réalité souvent déstabilisante du changement climatique.

Mais l'art ne peut pas s'enfermer dans des fantasmes stériles et des illusions cruelles. II se doit de répondre à de tels défis par l'image ou par les mots. La conscience écologique ne date pas d'hier, dans la culture française en particulier. De nombreux penseurs éclairés, au XVIIle et au XIXe siècle, de Rousseau à Fourier, avaient déjà ainsi exprimé la nécessité d'un retour à la nature dans le but de développer une plus grande humanité à l'intérieur d'un monde moderne soumis à la violence et aux rapports de domination et d'exploitation.

Le fameux 'Il faut cultiver son jardin' de Voltaire alla dans le même sens, celui d'une sagesse possible au milieu de la folie universelle. II faudrait alors parler d'écosophie, comme on parle de philosophie, et non pas simplement d'écologie, un terme qui est devenu trop commun et passe-partout. Le discours écologique, en effet, s'est aujourd'hui intégré dans les institutions 
et les partis politiques mainstream. II a perdu sa force de contradiction et de subversion, une force qu'il possédait à son origine, c'est-à-dire essentiellement dans les années soixante et soixante-dix, quand il participa d'une perspective contestataire générale sur la société bourgeoise et exprima un authentique projet contre-culturel. II fallait en ce sens, pour changer les rapports de domination à l'intérieur de la société qui s'incarnaient d'abord dans la lutte des classes, changer le rapport de l'homme à la nature, puisque ce rapport reposait lui-même sur une volonté de domination et de coercition.

Retrouver la nature, alors, c'était aussi se livrer sans retenue aux instincts, à l'éros et à la vie du corps, un choix existentiel que les penseurs radicaux en vogue des années soixante, de Marcuse à Wilhelm Reich, célébrèrent dans leurs écrits, qui constituaient une synthèse personnelle et ambitieuse de Marx et de Freud. L'écologie, dès lors, permettait idéalement de mettre fin au fameux 'malaise dans la civilisation' ${ }^{3}$.

Dans le monde intellectuel français, un penseur associé lui aussi à la radicalité des années soixante autant par ses ouvrages conçus en collaboration avec Deleuze, dont L'AntiCEdipe et Rhizomes, que par son travail de terrain dans le domaine des thérapies psychiatriques alternatives, se consacra à la fin de la vie à la question théorique de l'écologie, je veux parler de Félix Guattari dans son livre Trois Écologies, en particulier.

En ce sens, le discours écologique fondateur bouscula l'idée d'une existence sociale basée sur le cycle infernal de la production et de la consommation, et donc sur l'exploitation des ressources de la terre. II refléta une sensibilité hédoniste qui rejetait sans ambiguïté la souveraineté du principe de réalité dans l'organisation de l'existence sociale. A bien des égards,

\footnotetext{
3 Je me réfère ici à l'ouvrage désormais classique de Freud.

${ }^{4}$ Paris: Galilée, 1989.
} 
il affirma ainsi une identité qui était profondément incompatible avec l'ordre capitaliste et libéral. Se rapprocher de la nature, en effet, c'était aussi reconnaître l'importance de 'la part maudite' de l'homme, et donc de pulsions irrationnelles que la société cherchait trop souvent à contrôler.

Il faut bien avouer, en outre, que la présence de la conscience écologique à la fois dans le domaine politique et dans celui de l'art fut plus précoce en Allemagne fédérale qu'en France dans la seconde moitié du XXe siècle. Il suffit de songer à cet égard à l'émergence du mouvement des Verts (à laquelle d'ailleurs I'ancien leader de Mai 68, Daniel Cohn-Bendit, participa activement) outre-Rhin dès les années soixante.

On doit également se référer dans ce contexte à l'œuvre d'une figure majeure de l'art contemporain comme Joseph Beuys, dont l'engagement dans la sphère publique fut précisément issu d'une croyance profonde en le rapport éthique (et pas seulement esthétique) entre l'artiste et son environnement naturel. II fallut par contraste attendre les années quatrevingt en France pour assister à l'inclusion véritable du mouvement écologiste dans les divers gouvernements et pouvoirs de gauche, socialistes et communistes.

Un demi-siècle plus tard, cependant, le respect de la nature est devenu une idée fixe inscrite dans la vie quotidienne de la majorité des citoyens. La pensée de l'écologie a été largement récupérée par l'establishment politique qui a beau jeu, alors, de prétendre défendre les valeurs d'équilibre et de santé qu'elle implique nécessairement. L'écologie, selon cette perspective contemporaine, est devenue en quelque sorte l'un des meilleurs alibis du système néo-libéral, qui par ailleurs poursuit obstinément son projet économique et financier de 
destruction des services publics et des valeurs les plus fondamentales du contrat social en prétendant que la protection de l'environnement est parfaitement compatible avec celui-ci.

L'écologie ne fait (presque) plus peur à personne, en ce sens : elle a perdu une bonne part de sa signification communautaire originelle pour s'adapter aux valeurs individualistes contemporaines. Elle se réduit ainsi le plus souvent pour l'usager à un ensemble de mesures pratiques et à une gestion 'propre' de la vie quotidienne, sans que cette attitude perturbe réellement les rapports sociaux conventionnels issus de structures de pouvoir et de hiérarchies bien définies.

Le mouvement de Mai 68, au XXe siècle, réinterpréta à bien des égards le discours critique de Rousseau et surtout de Fourier, en montrant qu'il était indissociable d'une opposition radicale à la société bourgeoise et capitaliste et à son exigence permanente de rentabilité et de profit. N'oublions pas, dans cette optique, que Fourier fut le principal maître à penser de Breton après sa rupture officielle avec le communisme.

Il lui inspira une ode, L'Ode à Charles Fourier, qui demeure aujourd'hui encore l'un de ses textes poétiques les plus intéressants ${ }^{5}$. Au XXle siècle, cependant, notre rapport à la nature a changé dans la mesure où celle-ci n'est plus le lieu d'une utopie, comme c'était le cas manifestement avec Fourier et les étudiants de Mai 68 (ou les hippies de l'époque), mais bien une dystopie, compte tenu des catastrophes multiples évoquées plus haut.

Il nous faut donc repenser la nature, ce qui implique pour l'art de nouveaux modes de représentation. Dans cette optique, les paysages élégants et harmonieux des impressionnistes ne suffisent plus. Ils ne nous interrogent pas dans notre existence la plus crue et se contentent

\footnotetext{
${ }^{5}$ Paris: Librairie Klincksieck, 1961, avec une introduction de Jean Gaulmier.
} 
de flatter notre regard. On pourrait dans la même perspective évoquer l'art pourtant éminemment moderne d'un Cézanne. Les photographies de Terre brûlée, ainsi, ne participent en aucune manière de l'art du paysage, au sens classique du terme. Elles soulignent en effet par contraste l'absence du paysage, son vide quasi-définitif sous l'action du feu.

II n'y a plus rien à voir ni à contempler, en quelque sorte, sinon l'assaut des éléments contre la nature et ses formes. L'ancienne forêt royale s'est dans ce cas précis métamorphosée en une terre que la vie a abandonnée. Je me souviens qu'en observant pour la première fois avec stupeur un tel spectacle, je fus pris d'effroi et crut faire face à un paysage apocalyptique, proche de celui d'Hiroshima après l'explosion de la bombe atomique.

Le vide, pourtant, appelle encore une vision et une esthétique propre. II exige la présence de notre regard, un regard d'une grande acuité et attentif à l'ampleur de la destruction. Plus encore, il nous demande de dépasser la seule réalité pour imaginer l'après des évènements, ce qui pourrait advenir de cette nature saccagée par le feu. Ce qui m'avait le plus frappé, dans ce contexte, c'était la qualité de lumière venue du ciel qui contrastait avec la noirceur du sol.

Divers objets couverts de cendres, boîtes de conserve et cannettes, rappelaient également une présence humaine. Des nuages de fumée continuaient en outre à s'extraire de la terre plusieurs jours après l'extinction du feu. Je complétais alors mon travail de photographe par la composition d’un poème intitulé lui aussi Terre Brûlée :

Que reste-t-il à voir

Qu'est-ce qu'il y a là 
Du noir et encore du noir sous le ciel bleu

Un fin rayon de lumière entre les deux

Les arbres qui ne grandiront pas

Des traces de pas sur le sol

Des objets disséminés des squelettes faits de branches

Des vagues de feu un paysage nu

Une chaleur glaciale

Une crevasse qui s'étend

Un horizon dévasté

Une terre immobile

Une voix de l'au-delà creusant dans les racines

Rien qu'un espace dont on se souvient

Si loin de nous si proches de nos yeux

Les cendres d'hier

Une route qui mène à la mer

Une vérité enfouie qui respire à nouveau

Nous qui ne partirons jamais

Au-delà du vide, l'image peut ainsi insister sur un ensemble de traces qui persistent après la catastrophe. L'espace originel ne s'efface donc pas malgré le processus de dévastation incontestable. Il s'agit de montrer les forces à l'œuvre dans la nature et de comprendre ce qui nous lie malgré tout à celle-ci. Dans Terre brûlée, ce qui est fondamentalement en jeu, selon cette perspective, c'est la création d'une topo-poétique, c'est-à-dire un imaginaire 
essentiellement visuel du lieu qui s'incruste dans notre esprit longtemps après la réalité de l'évènement proprement dit.

Il faut pourtant reconnaître que la notion même de terre est aujourd'hui porteuse de nombreuses ambiguïtés. Elle n'est pas qu'une réalité physique, en effet, mais renvoie également à une pensée de l’appartenance qui a pu être récemment manipulée et exploitée par des forces politiques réactionnaires de type populiste. De la terre, ainsi, on passe vite au terroir et à l'idée d'un monde fixe et immuable qui ne se partage pas avec l'autre et ne tolère aucune différence.

Dans Terre Brûlée, par contraste, je veux insister sur la dimension essentiellement ouverte de cette terre. Elle n'appartient en quelque sorte à personne de particulier, et donc elle appartient à tout le monde. L'image de la destruction nous rassemble, en effet, au-delà de notre identité nationale ou culturelle. C'est qu'elle possède inévitablement un sens politique, surtout si l'on considère le nouveau négationnisme qui consiste aujourd'hui à ignorer les conséquences dramatiques du changement climatique.

La terre est bien ce lieu sur lequel nous vivons et qui nous fait vivre. Elle produit de nombreux fruits et engage notre esprit de façon constante. Mes images, dans cette perspective, rendent compte d'un processus de disparition et d'effacement qui ne peut que nous troubler. Ce processus nous arrache à nous-mêmes, en quelque sorte, à notre désir profond de paix et d'harmonie, mais aussi de liberté farouche. II évoque ainsi le sentiment d'une 'urgence climatique', selon l'expression choisie par l'Oxford Dictionary pour l'année 2019. La terre, en quelque sorte, ne peut pas attendre: elle exige une action commune et immédiate, 
car il y va de notre survie. On pourrait à cet égard parler de géo-existentialisme, soit d'une conscience aiguë de la force du présent issue de la terre et de ses multiples bouleversements.

Cette terre possède en outre simultanément une dimension globale ou universelle et une dimension particulière ou singulière. Globale, parce qu'elle nous concerne tous en tant que citoyens du monde épris de contact avec la nature et de préservation de celle-ci, au-delà de toutes les frontières concrètes. Mais elle est aussi inscrite dans mon espace personnel, intérieur et imaginaire, celui que j'invente jour après jour pour mieux me définir moi-même.

La terre, dans cette perspective, se reconstruit et se recompose sans cesse selon mes désirs et selon mes rêves. Je cherche alors à en faire une œuvre d'art en soulignant la beauté de ses formes, quels que soient les assauts et les violences dont elle est l'objet. Il s'agit en ce sens de saisir une proximité essentielle de cette terre à tout moment, qui est d'ordre psychique et spirituel avant d'être géographique.

Les mouvements radicaux des années soixante que j'ai évoqués auparavant soulignèrent ainsi l'identité poétique de cette terre soumise à notre regard et à nos besoins de transcendance. Ils en révélèrent l'aspect utopique, celui d'un lieu situé à la fois près et loin de nous. La catastrophe transforme ainsi ce lieu en un site authentique, comme on parle du site du onze septembre, à New York, par exemple. Le site possède inévitablement une signification historique. II exige un travail de mémoire qui se déroule dans le temps et permet de conserver en nous la représentation nécessaire de la destruction pour mieux alors la contredire et la dépasser.

Le parti-pris esthétique du vide, dans cette optique, témoigne d'une lutte contre l'absence. L'homme est toujours visible, en effet, au milieu de l'espace naturel aussi désert et 
nu soit-il en apparence. Il s'agit sans doute d'un homme qui reste à inventer ou à parfaire, mais peu importe. Son ombre se profile sans cesse à l'horizon du néant, en tant que possibilité imaginaire qui mène à une nouvelle relation plus équilibrée avec la nature. C'est l'art alors qui éclaire le mieux cette promesse d'équilibre à venir, quelles que soient les sombres circonstances qui lui ont donné le jour. 
